\title{
miR-187 inhibits tumor growth and invasion by directly targeting MAPK12 in osteosarcoma
}

\author{
CHENGLIANG $\mathrm{CUI}^{1 *}$ and XIAOYU $\mathrm{SHI}^{2 *}$ \\ ${ }^{1}$ Department of Orthopedics, The First Affiliated Hospital of PLA General Hospital (304 Hospital), Beijing 100048; \\ ${ }^{2}$ Department of Medical Oncology, The First Affiliated Hospital of Hebei \\ North University, Zhangjiakou, Hebei 075000, P.R. China
}

Received December 15, 2016; Accepted June 2, 2017

DOI: $10.3892 /$ etm.2017.4624

\begin{abstract}
MicroRNAs (miRNAs/miRs) play crucial roles in cancer development and progression. The purposes of this study were to investigate the role of miR-187 in osteosarcoma and clarify the regulation of MAPK12 by miR-187. Quantitative polymerase chain reaction was used to examine miR-187 expression in osteosarcoma tissues and cell lines. The clinicopathological significance of miR-187 downregulation was further analyzed. Transwell migration and invasion assays were performed. A luciferase reporter assay was conducted to confirm the target gene of miR-187, and the results were validated in cervical cancer tissues and cell lines. MiR-187 was significantly decreased in clinical tissues and osteosarcoma cell lines. The low miR-187 level was significantly correlated with stage, node metastasis, and deep stromal invasion. Upregulation of miR-187 suppressed cell migration and invasion in vitro. MAPK12 was verified as a direct target of miR-187, which was further confirmed by the inverse expression of miR-187 and MAPK12 in patients' specimens. The newly identified miR-187/MAPK12 pathway provides an insight into osteosarcoma metastasis and may represent a novel therapeutic target.
\end{abstract}

\section{Introduction}

Osteosarcoma is one of the most common primary bone malignancies and is the third most frequent cancer in adolescentsand young adults (1). Osteosarcoma localizes to the proximal tibia or the distal femur and has a highly malignant tendency to invade the surrounding normal tissues and to

Correspondence to: $\mathrm{Dr}$ Chengliang Cui, Department of Orthopedics, The First Affiliated Hospital of PLA General Hospital (304 Hospital), 51 Fucheng Road, Beijing 100048, P.R. China E-mail: cuichengliangchina@126.com

*Contributed equally

Key words: osteosarcoma, miR-187, MAPK12, metastasis metastasize. Usually, the treatments for osteosarcoma are chosen as surgical resection, chemotherapy and radiotherapy, but the prognosis remains poor because of aggressive invasion into local tissue and extremely-high metastatic rate. Hence, novel therapeutic approaches, such as target therapies against specific targets, are urgently required.

MicroRNAs (miRNAs/miRs) are a class of small noncoding and endogenous regulatory RNA molecules that are approximately 19-25 nucleotides in length, which can bind the 3'-untranslated region (3'-UTR) of specific genes to inhibit mRNA translation then impair the protein process, act as posttranscriptional regulations of gene expression (2). Micro RNAs can regulate the biological function in cancer cells, be involved in a variety of critical cellular processes, including cell differentiation, proliferation and metabolism. Besides, some miRNAs has been used to classify human cancers and markers for predicting the prognosis $(3,4)$.

MiRNA play a significant role in tumor progression, including tumorigenesis, invasion, metastasis and angiogenesis in malignant tissues, such as osteosarcoma. They can regulate the cell function through tumor suppressors and oncogenes in osteosarcoma, such P53 (5), SPRED-1 (6), and BCL-2 $(7,8)$. He et al (5) found that miR-34 family as direct transcriptional targets of p53 tumor suppressor, which regulate the DNA damage, cellular stress and improper cell proliferation. Moreover, they found that ectopic expression of miR-34 induces cell cycle arrest across osteosarcoma cells and cell lines. Maire et al (6) found that miR-126 is overexpressed in human osteosarcoma specimens. miR-126 has been reported to repress SPRED-1, which is an inhibitor of mitogen-activated protein kinase (MAPK) signaling, and stimulate vascular endothelial growth factor (VEGF)-induced angiogenesis, to enhance cell migration, proliferation and survival. Therefore, the miRNA play a significant role in osteosarcoma development.

Recent study showed that miR-187 is significantly downregulated in many types of human malignancies, including renal carcinoma, pancreatic cancer, thyroid cancer, gastric cancer, esophageal cancer, prostate cancer, breast cancer, ovarian cancer, and lung carcinomas (9-18). The biological functions were controversial to date. In breast cancer, miR-187 could leads to a more aggressive, invasive cancer type and had a worse prognosis (16). In pancreatic cancer, miR-187 can predict short overall survival (OS) in patients after radical 
surgery. In colorectal cancer, the miR-187 could inhibit the tumor growth and invasion (19). To date, the differences of miR-187 function in different cancers has not been clear.

In this study, we studied the miR-187 expression in osteosarcoma tissues and cell lines, then investigated its functions on osteosarcoma cell migration and invasion after transfected miR-187 mimic plasmids. Furthermore, we explored that MAPK12 was the direct target for miR-187 in osteosarcoma cells and was involved in the functional influence of miR-195 on osteosarcoma cells migration and invasion.

\section{Materials and methods}

Patients and specimens. The human clinical samples were collected at 304 hospitals surgical specimens. The study was approved by the ethical board of the hospital. All samples were collected in liquid nitrogen for a quick frozen and then stored at $-80^{\circ} \mathrm{C}$ until RNA extraction.

Cell culture and transfections. The human osteosarcoma cancer cell line, U2OS cells was cultured in DMEM supplemented with $10 \% \mathrm{FBS}$ at $37^{\circ} \mathrm{C}$ in $5 \% \mathrm{CO}_{2}$.

miR-187 mimicsplasmids, MAPK12 siRNA, and scramble control were ordered from Jima company and transfected into osteosarcoma cells with Lipofectamine ${ }^{\circledR} 3000$ following protocols.

Cell proliferation assay. The transfected cells were incubated with $10 \% \mathrm{CCK}-8$ at $37^{\circ} \mathrm{C}$ for $2 \mathrm{~h}$ then measured at $450 \mathrm{~nm}$ absorption for examining the effect of cell proliferation rate. Proliferation rates were detected at days 1,2,3, 4 and 5 after plasmids and siRNAs transfection. The proliferation rates then were calculated with software.

Cell migration and invasion assays. After transfected, osteosarcoma cells were seeded onto a Transwell and Matrigel chamber inserting into a well of a 24-well plate in serum-free medium. $10 \%$ FBS was added to bottom chamber. The seeded cells was counted as $1 \times 10^{5}$ each chamber. After $24 \mathrm{~h}$, migrated and invasive cells on the lower surface of the chamber were stained with $0.1 \%$ crystal violet and counted.

RNA extraction and real-time PCR assays. Total RNA was extracted using TRIzol from tissues and cells according to the manufacturer's instructions. Quantitative PCR was performed on a Bio-Rad CFX96 Real-Time PCR System with TaqMan probes. The PCR conditions were as follows: $95^{\circ} \mathrm{C}$ for $30 \mathrm{sec}$, followed by 42 cycles of $95^{\circ} \mathrm{C}$ for $5 \mathrm{sec}$, and $58^{\circ} \mathrm{C}$ for $35 \mathrm{sec}$. MAPK12 primes forward: 5'-CCACCTTCACCTTCCACC T-3'. Reverse: 5'-GCGTCTGCTCTGATGGATG-3'.

Luciferase assay. For the dual luciferase assay, U2OS cells in a 96-wells plate were transfected with miR-195 or miRNA negative control. The cells were then cotransfected with $0.2 \mathrm{mg} / \mathrm{ml}$ of vector with the 3'-UTR of Smad7 gene. After $48 \mathrm{~h}$, luciferase activity was measuredwith the Dual-Luciferase reporter assay system (Promega Corp., Madison, WI, USA). Firefly luciferase activity was then normalized to the corresponding Renilla luciferase activity. Luciferase assays were performed in quadruplicate and repeated in 3 independent experiments.
Table I. Correlation of Mir-187 expression with clinical pathological parameters of patients with Osteosarcoma.

\begin{tabular}{lcrrr}
\hline & & miR-187 expression & \\
& No. of & & \\
& patients & (Low) & (High) & P-value \\
\hline Age (years) & & & & 0.2165 \\
$>20$ & 20 & 12 & 8 & \\
$\leq 20$ & 22 & 9 & 13 & \\
Sex & & & & 0.0213 \\
Male & 29 & 18 & 11 & \\
Female & 13 & 3 & 10 & \\
Clinical stage & & & & 0.0023 \\
I-II & 22 & 6 & 16 & \\
III-IV & 20 & 15 & 5 & \\
Histologic grade & & & & 0.0002 \\
I-II & 20 & 4 & 16 & \\
III-IV & 22 & 17 & 5 & \\
Metastasis & & & & 0.0042 \\
Yes & 10 & 9 & 1 & \\
No & 32 & 12 & 20 & \\
\hline & & & & \\
\hline
\end{tabular}

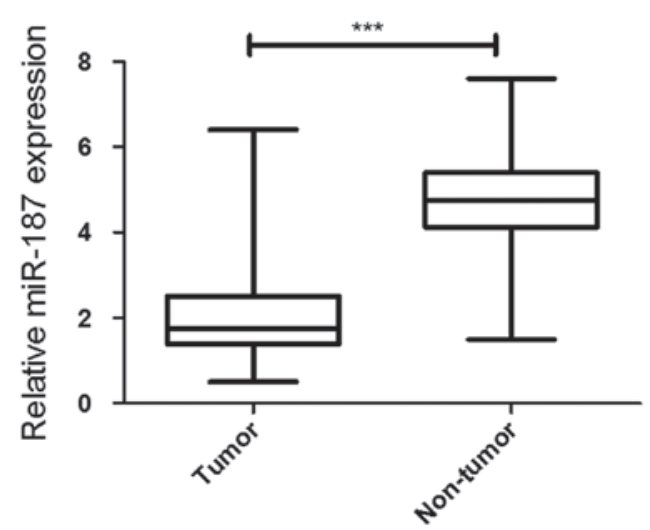

Figure 1. The mean expression level of miR-187 in osteosarcoma tissues was significantly lower than that in pair-matched adjacent normal tissues $\left({ }^{* * * *} \mathrm{P}<0.001\right)$.

Western blotting. Cells were collected and lysed in ice-cold RIPA buffer (Beyotime Biotech, Jiangsu, China) according to the manufacturer's instructions. Protein concentration was quantified using an Enhanced BCA protein assay kit (Beyotime Biotech). The proteins were separated by sodium dodecyl sulfate polyacrylamide gel electrophoresis, and transferred onto polyvinylidene difluoride membranes (Millipore Corp., Bedford, MA, USA). After being blocked with $5 \%$ free-fat milk for $1.5 \mathrm{~h}$ at room temperature, membranes were incubated with anti-MAPK12 antibody (Cell Signaling Technology, Inc., Danvers, MA, USA; 1:1,000 dilution overnight at $4^{\circ} \mathrm{C}$, followed by incubation with horseradish peroxidase $\mathrm{Y}$ conjugated mouse and rabbit secondary antibody (Beyotime Biotech) for $2 \mathrm{~h}$ at room temperature. 

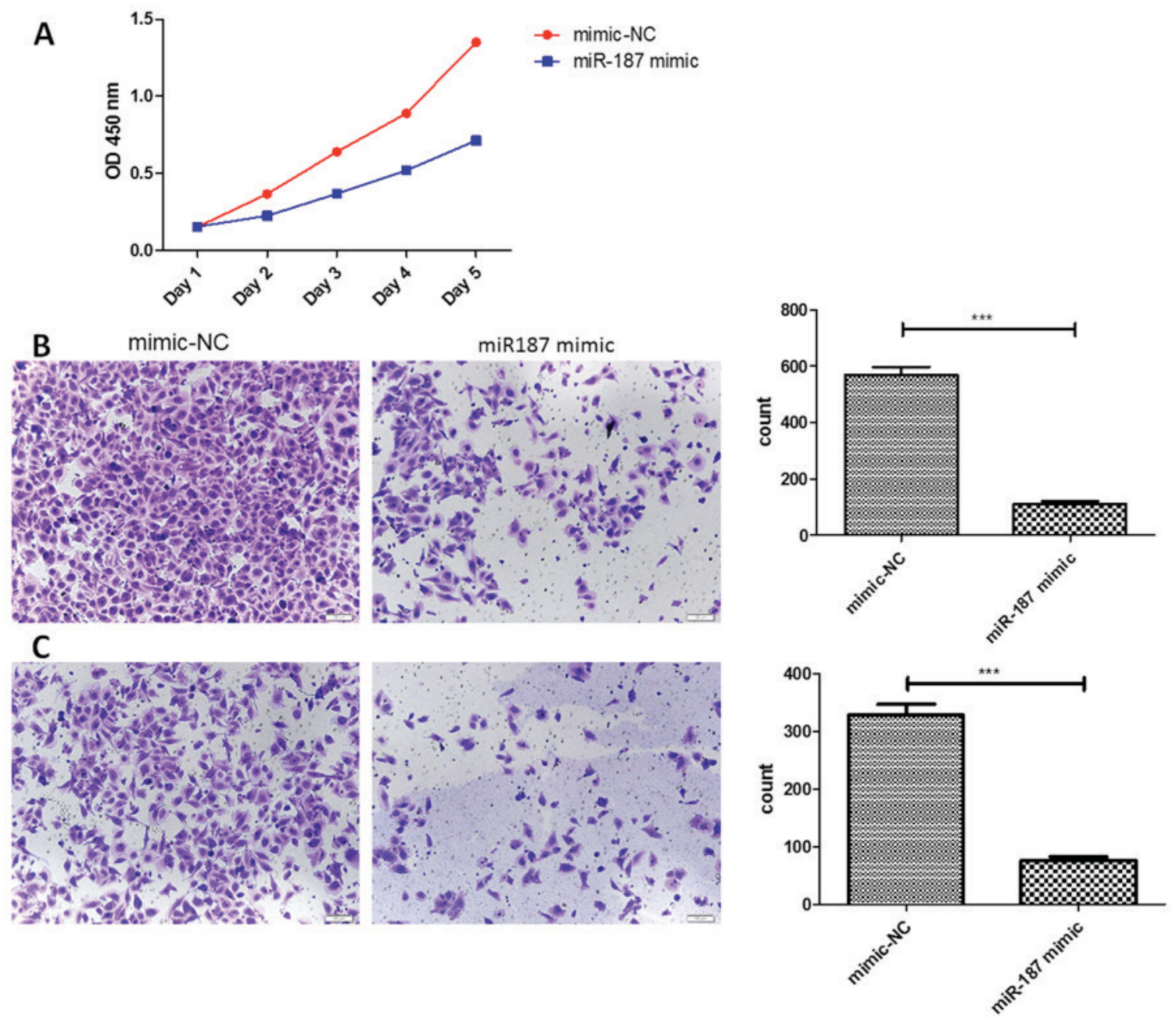

Figure 2. (A) CCK-8 assay of U2OS cells. (B) Analysis the effect of miR-187 on the migration of U2OS cells by Transwell migrationassay. (C) Matrigel invasion assay was used to analyze the effect of miR-187 on the cell invasion of U2OS cells. The data represent the mean values of 3 independent experiments. ${ }^{* * * *} \mathrm{P}<0.001$. Scale bar $=100 \mu \mathrm{m}$.

Statistics. Student's t-test (two tailed) was performed to analyze the data. Values of $\mathrm{P}<0.05$ were considered significantly.

\section{Results}

miR-187 expression is significantly downregulated in osteosarcoma tissues and cell lines. To analyze 42 paired tumor and adjacent non-tumor tissue samples in 304 hospital during 2011-2015, the miR-187 was significantly decreased in the osteosarcoma by using quantitative PCR. Meanwhile, miR-187 expression was significantly decreased in the in cell lines compared with that of U2OS cells (Fig. 1).

MiR-187 expression was as potential marker for diagnosis and prognosis to the osteosarcoma. The relation between miR-187 expression level and clinical parameters was further analyzed in 42 osteosarcoma patients. The statistical analysis showed that lower miR-187 expression level was significantly correlated with pathological grading and distant metastasis. (Table I). These results suggested that the miR-187 downregulation maybe involved in the invasion and metastasis of osteosarcoma (Table I).
miR-187 could suppress the osteosarcoma cells proliferation, migration and invasion. The miR-187 was found significantly decreased in the osteosarcoma cells, then we investigated the miR-187 biological function in osteosarcoma cells. After transfected in miR-187 mimics plasmids, we found the difference of biological behavior with the corresponding negative control. In CCK-8 assay, we discovered the proliferation ability of osteosarcoma cells was affected. We found that the proliferation rate was lower in the miR-187 mimics groups, especially in the 4th day. Besides, the Transwell assay showed that the miR-187 mimics groups had a less cells in the migrated sphere, suggested the miR-187 could suppress the migration in the osteosarcoma cells. In the Matrigel assay, the miR-187 mimics group also showed the less cells migrated, suggested the miR-187 could suppress the invasiveness in the osteosarcoma cells (Fig. 2).

MAPK12 was the direct target of $m i R-187$. To discover the mechanism of MiR-87 affecting the biological behavior in osteosarcoma cells, we constructed luciferase reporter vector containing MAPK12 3'-UTR. Luciferase activity assay demonstrated that miR-187 significantly inhibited the luciferase activity of MAPK12-3'-UTR reporter comparing with control group (Fig. 3). 
A

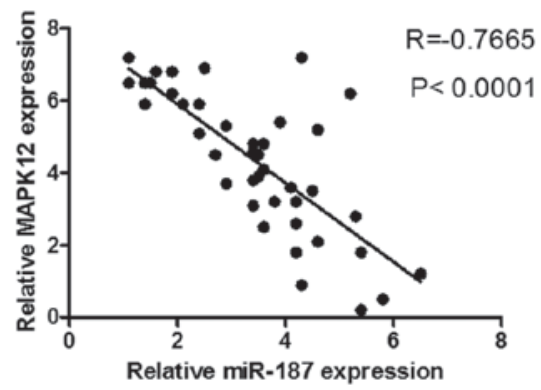

B Predicted binding sites of miR-187 on $3^{\prime}$-UTR of MAPK12 mRNA

MAPK12 on 3'-UTR(488-494)

$5^{\prime}$

U CGAUGUUUUCCCAG
C

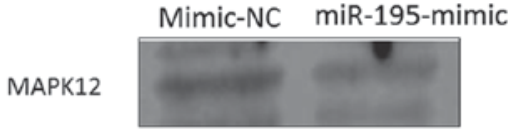

$\beta$-actin

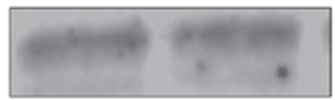

D

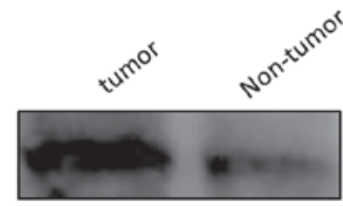

$3^{\prime}$

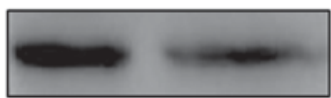

3

Figure 3. MAPK12 is a direct target of miR-187. (A) Putative miR-187 binding site in 3'-UTR region of MAPK12 and interspecies conservation of seed matching sequences. (B) Smad3 mRNA level was inversely correlated with miR-187 level in osteosarcoma tissues (Spearman correlation analysis). (C) Protein level of Smad3 was detected by western blotting in U2OS cells transfected with miR-187 mimics along with corresponding controls. (D) Protein level of MAPK12 in osteosarcoma tissues and normal tissues by western blotting.

A

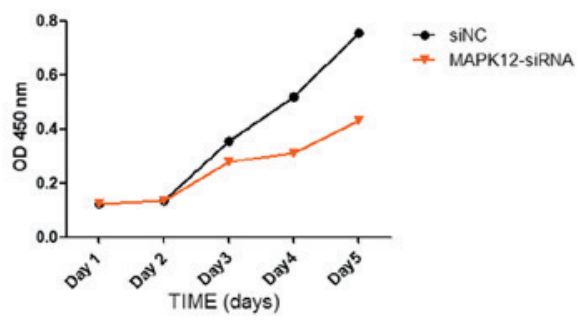

B
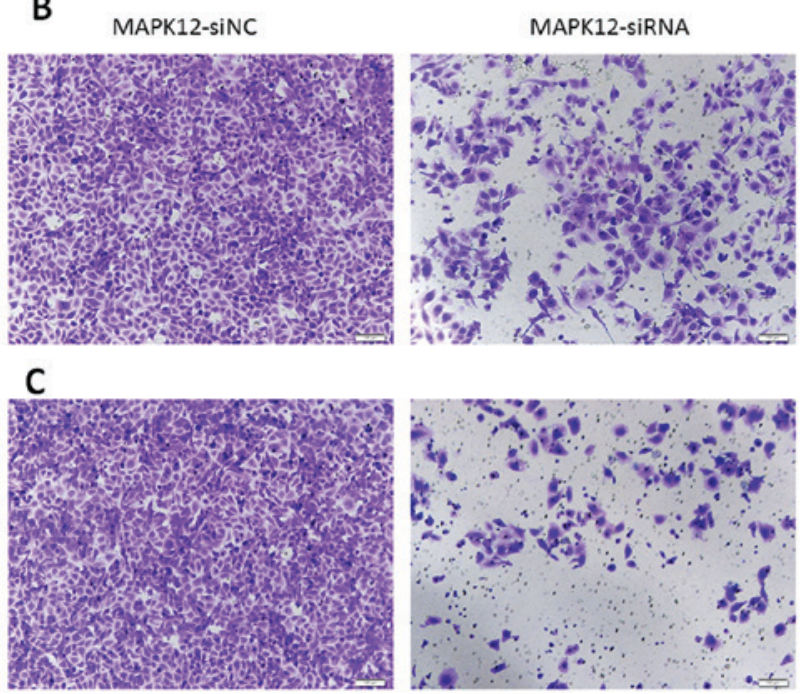

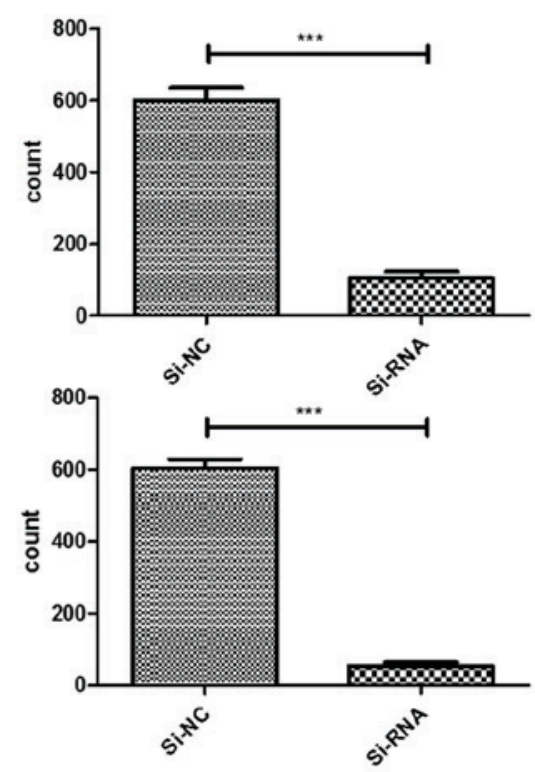

Figure 4. Knockdown of MAPK12 with siRNA. (A) CCK-8 assay after knockdown of MAPK12. The knockdown of MAPK12. (B) Analysis the effect of knockdown of MAPK12 through Transwell. (C) Analysis the effect of knockdown of MAPK12 through Matrigel. Scale bar=100 $\mu \mathrm{m}$.

Knockdown of MAPK12 can inhibit osteosarcoma cell proliferation, migration and invasion. To investigate the role of MAPK12 in osteosarcoma progression, we knocked down
MAPK12 expression by siRNAs in two osteosarcoma cell lines. Knockdown of MAPK12 attenuated osteosarcoma cell migration significantly as demonstrated by CCK-8, Transwell 
and Matrigel assay, suggested MAPK12 may play an important role in the osteosarcoma cells proliferation, migration and invasion. The aberrant downregulation of miR-187 in osteosarcoma may give rise to upregulation of MAPK12 and further to promote osteosarcoma cancer progression (Fig. 4).

\section{Discussion}

In this study, we explored the miR-187 expression in osteosarcoma tissues and cells, and results showed that the miR-187 was decreased in the both osteosarcoma tissues and cells. After transfected the miR-187 mimics plasmids, we found that the osteosarcoma cells proliferation, migration and invasion ability was inhibited. The Luciferase activity assay demonstrated that miR-187 targeted the MAPK12 for regulating the cell biological function. Knockdown MAPK12 could inhibit the proliferation and migration ability of osteosarcoma cells.

Several studies have investigated the association between aberrant miR-187 expression and the risk of diverse cancers. Emerging evidence has shown that aberrant expression of miRNAs contributes to tumorigenesis and potentiallyservesas biomarkers for prediction and prognosis in various cancers including osteosarcoma. Recently, miR-187 was found downregulated in cancer tissues, such as thyroid cancer, gastric cancer, esophageal cancer, prostate cancer, breast cancer, ovarian cancer, and lung carcinomas $(11,14-16,19,20)$. In this study, we further confirmed that miR-187 can be downregulated in the osteosarcoma. Osteosarcoma is a severe disease that occurs in a very young age and with a bad prognosis. Despite of conventional therapy, many patients still suffers with a very poor prognosis. Therefore, new therapy and early diagnosis methods should be developed for an in-time diagnosis. In several studies, the miR-187 expression was a marker for some malignant tumor, such as miR-187 playing oncogenic roles in oral carcinogenesis and Plasma miR-187 could be validated as a marker of OSCC for diagnostic uses. In this study, we found that miR-187 expression level was significantly correlated with pathological grading and distant metastasis, suggested that the miR-187 may be the suitable marker for predicting the prognosis in the future.

Moreover, we found that miR-187 could suppress the ostrosacoma cells proliferation. The results suggested miR-187 maybe act as a tumor suppressor in ostrosacoma. Downregulation or deletion of miR-187 may initiate the oncology activity. In this study, we found that the proliferation was inhibited in mimic-187 group, so we redid the cell counts to make sure there being same cell population in both control group and mimic-187 group before planting cells in the upper chamber of Transwell and Matrigel assays. We did this part to avoid the influence of the proliferation. The results showed that miR-187 could suppress the ostrosacoma cells migration and invasion. However, some studies showed that the miR-187 also promote the migration and proliferation in other cancer cells, suggested that the miR-187 may affect the biological behavior in specific cancers.

To date, some studies showed that CD276, BCL6 was the target of miR-187 (19). In this study, we also found that the MAPK12 was a target of miR-187. MAPK12 has been shown to regulate cancer cell metastases in some types of cancer. MAPK11 and MAPK12 are two of the four p38 MAPKs that play important roles in the cellular responses evoked by extracellular stimuli, and MAPK12 plays a role in repressing cell proliferation through the downregulation of cyclin D1 in response to hypoxia in adrenal cells. In this study, downregulating the MAPK-12 could suppress the proliferation, migration and invasiveness in ostrosacoma cells, which verified that MAPK12 promotes the biological behavior in the ostrosacoma cells. And we also confirmed that MAPK12 was a direct target of miR-187, which expanded the potential target network of miRNA-187. Although we identified miR-195 maybe act as a direct regulator of MAPK12 expression, we could not conclude that MAPK12 was only regulated by miR-187, there was the possibility of modification of MAPK12 protein levels through miRNAs other than miR-187, which may be found in future studies. Besides, as discussed above, miR-187 also regulate the cell cycle to regulate cancer cell growth. These data reflect the complexity of the regulation of tumorigenesis by a network of molecules in cancers with micro RNAs.

In conclusion, we found that miR-187 was decreased in the ostrosacoma tissues and cells, which had clinical relationships with advantage stages and metastasis. The miR-187 suppressed the proliferation, migration and invasion by targeting the MAPK12.

\section{References}

1. He JP, Hao Y, Wang XL, Yang XJ, Shao JF, Guo FJ and Feng JX: Review of the molecular pathogenesis of osteosarcoma. Asian Pac J Cancer Prev 15: 5967-5976, 2014.

2. Flynt AS and Lai EC: Biological principles of microRNA-mediated regulation: Shared themes amid diversity. Nat Rev Genet 9: $831-842,2008$.

3. Friedman RC, Farh KK, Burge CB and Bartel DP: Most mammalian mRNAs are conserved targets of microRNAs. Genome Res 19: 92-105, 2009.

4. Griffiths-Jones S, Saini HK, van Dongen S and Enright AJ: miRBase: Tools for microRNA genomics. Nucleic Acids Res 36 (Database issue): D154-D158, 2008.

5. He L, He X, Lim LP, de Stanchina E, Xuan Z, Liang Y, Xue W, Zender L, Magnus J, Ridzon D, et al: A microRNA component of the p53 tumour suppressor network. Nature 447: 1130-1134, 2007.

6. Maire G, Martin JW, Yoshimoto M, Chilton-MacNeill S, Zielenska $M$ and Squire JA: Analysis of miRNA-gene expression-genomic profiles reveals complex mechanisms of microRNA deregulation in osteosarcoma. Cancer Genet 204: 138-146, 2011.

7. Aqeilan RI, Calin GA and Croce CM: miR-15a and miR-16-1 in cancer: Discovery, function and future perspectives. Cell Death Differ 17: 215-220, 2010.

8. Xia L, Zhang D, Du R, Pan Y, Zhao L, Sun S, Hong L, Liu J and Fan D: miR-15b and miR-16 modulate multidrug resistance by targeting BCL2 in human gastric cancer cells. Int J Cancer 123: 372-379, 2008.

9. Gu L, Li H, Chen L, Ma X, Gao Y, Li X, Zhang Y, Fan Y and Zhang X: MicroRNAs as prognostic molecular signatures in renal cell carcinoma: A systematic review and meta-analysis. Oncotarget 6: 32545-32560, 2015.

10. Bloomston M, Frankel WL, Petrocca F, Volinia S, Alder H, Hagan JP, Liu CG, Bhatt D, Taccioli C and Croce CM: MicroRNA expression patterns to differentiate pancreatic adenocarcinoma from normal pancreas and chronic pancreatitis. JAMA 297: 1901-1908, 2007.

11. Nikiforova MN, Tseng GC, Steward D, Diorio D and Nikiforov YE: MicroRNA expression profiling of thyroid tumors: Biological significance and diagnostic utility. J Clin Endocrinol Metab 93: 1600-1608, 2008.

12. Lo SS, Hung PS, Chen JH, Tu HF, Fang WL, Chen CY, Chen WT, Gong NR and $\mathrm{Wu} \mathrm{CW}$ : Overexpression of miR-370 and downregulation of its novel target TGF $\beta$-RII contribute to the progression of gastric carcinoma. Oncogene 31: 226-237, 2012. 
13. Wijnhoven BP, Hussey DJ, Watson DI, Tsykin A, Smith CM and Michael MZ; South Australian Oesophageal Research Group: MicroRNA profiling of Barrett's oesophagus and oesophageal adenocarcinoma. Br J Surg 97: 853-861, 2010.

14. Casanova-Salas I, Masiá E, Armiñán A, Calatrava A Mancarella C, Rubio-Briones J, Scotlandi K, Vicent MJ and López-Guerrero JA: MiR-187 targets the androgen-regulated gene ALDH1A3 in prostate cancer. PLoS One 10: e0125576, 2015.

15. Blenkiron C, Goldstein LD, Thorne NP, Spiteri I, Chin SF Dunning MJ, Barbosa-Morais NL, Teschendorff AE, Green AR, Ellis IO, et al: MicroRNA expression profiling of human breast cancer identifies new markers of tumor subtype. Genome Biol 8 : R214, 2007.

16. Mulrane L, Madden SF, Brennan DJ, Gremel G, McGee SF, McNally S, Martin F, Crown JP, Jirström K, Higgins DG, et al: miR-187 is an independent prognostic factor in breast cancer and confers increased invasive potential in vitro. Clin Cancer Res 18 6702-6713, 2012.
17. Chao A, Lin CY, Lee YS, Tsai CL, Wei PC, Hsueh S, Wu TI, Tsai CN, Wang CJ, Chao AS, et al: Regulation of ovarian cancer progression by microRNA-187 through targeting Disabled homolog-2. Oncogene 31: 764-775, 2012.

18. Li X, Shi Y, Yin Z, Xue X and Zhou B: An eight-miRNA signature as a potential biomarker for predicting survival in lung adenocarcinoma. J Transl Med 12: 159, 2014.

19. Wang ZS, Zhong M, Bian YH, Mu YF, Qin SL, Yu MH and Qin J: MicroRNA-187 inhibits tumor growth and invasion by directly targeting CD276 in colorectal cancer. Oncotarget 7: 44266-44276, 2016.

20. Schultz NA, Andersen KK, Roslind A, Willenbrock H, Wøjdemann M and Johansen JS: Prognostic microRNAs in cancer tissue from patients operated for pancreatic cancer-five microRNAs in a prognostic index. World J Surg 36: 2699-2707, 2012. 